\title{
RANDOM SETS AND CONFIDENCE PROCEDURES
}

\author{
WILLIAM A. BARNETT*
}

\begin{abstract}
The set of confidence procedures is identified as a fundamental subset of the set of random sets. This is accomplished by defining a simple $\sigma$-field on the codomain of a set-valued mapping such that the mapping is a confidence procedure if it is measurable relative to that $\sigma$-field. The confidence procedure becomes Borel measurable when the $\sigma$-field is generated by a topology that we also define. The resulting topology and $\sigma$-fields are shown to be natural choices on $\mathscr{P}\left(R^{n}\right)-\{\not\}$. The motivation for the approach is the same as that motivating the use of statistics in point estimation. The theory of confidence procedures in set estimation is shown to be a simple extension of the theory of statistics in point estimation.
\end{abstract}

1. Introduction. Confidence sets are defined to be realizations of random sets called confidence procedures. Hence the random sets of set estimation correspond naturally to the random variables of point estimation. While the simple definition of confidence sets used in elementary applied statistics suffices to describe those set-valued mappings relevant to elementary statistics, a definition of confidence sets appropriate to measure-theoretic formulations should characterize confidence procedures in general. The purpose of this paper is to provide such a characterization and to illustrate that the resulting confidence procedures are particularly simple and fundamental measurable set-valued mappings. The method adopted presents the theories of confidence procedures and random sets as extensions to the theories of statistics and random variables.

A step in the direction followed by this paper was taken by Wallace [13], who characterized a subset of the set of confidence procedures in terms of the properties of the mappings' graphs. Joshi's ([3], [4], [5], [6], [7]) recent work investigating the admissibility of common confidence procedures has demonstrated the usefulness of Wallace's definition in systematic studies of confidence procedures. Similarly Stein [12] has

Received by the editors on September 4, 1975, and in revised form on March 12,1977.

*This research was initiated in the Department of Statistics at Carnegie-Mellon University. Completion of the research was supported by the Board of Governors of the Federal Reserve System in Washington, D.C. The author would like to thank Professor Paul Shaman and one of the Editors for their helpful comments.

AMS(MOS) 1970 Subject classifications: 28A05, 62F25, 54A05.

Key words and phrases: neighborhood system topology, confidence procedures, random sets. 
used Wallace's definition in the investigation of confidence procedures for the mean of the multivariate normal distribution. Nevertheless, no direct and complete characterization of the set of confidence procedures as random sets appears to exist. Although not directly relevant to our objectives, general discussions of the mathematics of random sets and of topologies on power sets are available in Kendall [8], Matheron [9], and Michael [10]. Well known mathematical results used freely throughout our proofs are conveniently available in [1], [2], and [11].

2. A Simple Class of Random Sets. This section will begin with a summary of the relevant types of mappings. Let $(\Omega, \mathscr{A}, \mu)$ be a measure space with $\sigma$-field $\mathscr{S}$ and measure $\mu$, and let $(Y, \mathscr{F})$ be an abstract measurable space with $\sigma$-field $\mathscr{F}$. Then $f:(\Omega, \mathscr{A}) \rightarrow(Y, \mathscr{F})$ is a measurable transformation if $f^{-1}(\mathscr{F}) \subset \mathscr{A}$, where $f^{-1}$ is defined to be the inverse mapping induced by $f$, such that $f^{-1}\left(Y_{0}\right)=\left\{\omega \in \Omega: f(\omega) \in Y_{0}\right\}$ for every $Y_{0} \subset Y$. We shall say that $Q: \mathscr{F} \rightarrow R$ is the distribution of $f$ (or measure induced on $(Y, \mathscr{F})$ by $f$ ) if $Q(F)=\mu[f \in F]$ for every set $F \in \mathscr{F}$.

Let $\mathscr{P}\left(R^{n}\right)$ be the power set of $R^{n}$. Then if $f$ is a measurable transformation and if $Y \subset \mathscr{P}\left(R^{n}\right)-\{\phi\}, f$ will be called a measurable correspondence. Finally $f$ will be called a random set if it is a measurable correspondence and if $\mu$ is a probability measure.

Let $\operatorname{Pr}$ be a probability measure on $(\Omega, \mathscr{A})$ and let $f:(\Omega, \mathscr{A}, \operatorname{Pr}) \rightarrow$ $(Y, \widetilde{F})$ be a random set. For a random variable, the usual $\sigma$-field on its codomain is the Borel field ( $\sigma$-field generated by the topology on a topological space) generated by the ordinary topology on $R$. To give the measurability of $f$ a similarly natural meaning, a simple topology on $Y$ will be sought to generate an elementary Borel field on $Y$. That Borel field will be $\mathscr{F}$. We also shall consider the selection of a $\sigma$-field $\mathscr{F}$ on $Y$ when the measurability of $f$ need not be Borel.

Let $T$ be the discrete topology on $R^{n}$, and let $\mathcal{N},-(z)$ be the neighborhood system of $z \in R^{n}$ in the topological space $\left(R^{n}, T\right)$. Then $\mathcal{N}, \pi(z)$ $=\left\{U \subset R^{n}: z \in U\right\}$. If $\mathcal{N}_{T^{*}}$ is defined to be the set function induced on $R^{n}$ by $\mathcal{N}_{T}$, then $\mathcal{N}_{T^{*}}\left(R^{n}\right)=\left\{\mathcal{N}_{,}(z): \mathrm{z} \in R^{n}\right\}$ is the range of the point function $N^{\prime}$ and is the collection of all neighborhood systems in $\left(R^{n}, T\right)$. Viewing $N^{\prime}, T^{*}\left(R^{n}\right)$ as a class of elementary sets on $\mathscr{P}\left(R^{n}\right)-\{\Phi\}$, the following simple topologies can be defined.

DEFINITION 1. The neighborhood system topology on $\mathscr{P}\left(R^{n}\right)-\{\phi\}$ is the topology generated by the subbase $N^{\prime} \Omega^{*}\left(R^{n}\right)$.

Definition 2. The subspace neighborhood system topology on $Y$ is the relative topology induced on $Y$ by the neighborhood system topology on $\mathscr{P}\left(R^{n}\right)-\{\varnothing\}$. 
We now shall define a $\sigma$-field on $Y$.

Definition 3. The elementary Borel field on $Y$ is the $\sigma$-field generated by the subspace neighborhood system topology on $Y$.

Let $\Theta \subset R^{n}$ such that $\cup Y \subset \theta$, let $C_{\theta}=\mathcal{N}^{\prime}(\theta) \cap Y$ for every $\theta \in \theta$, and let $\Sigma=\left\{\mathscr{C}_{\theta}: \theta \in \theta\right\}$. Then by a simple property of relative topologies, the subspace neighborhood system topology on $Y$ is equal to the topology on $Y$ generated by $\Sigma$. We shall say that a Borel field is generated by a collection of sets, $\mathcal{D}$, if the Borel field is generated by the topology having $\eta$ as subbase. Then the elementary Borel field on $Y$ is the Borel field generated by $\Sigma$. This observation reveals the relationship between Definition 3 and the following definition, in which we use $\sigma(\Sigma)$ to denote the $\sigma$-field generated by $\Sigma$.

Definition 4. The elementary $\sigma$-field on $Y$ is $\sigma(\Sigma)$.

Then if $\mathscr{F}$ is the elementary $\sigma$-field on $Y$, the $(\mathscr{F}, \mathscr{F})$-measurable random sets will be seen below to be to measurable correspondences what statistics are to measurable functions. As we shall see in a later section, this analogy becomes even stronger when $\mathscr{F}$ is the elementary Borel field on $Y$.

3. Statistics and Estimation. To motivate our theory of set estimation, we shall digress in this section with a review of the analogous and well established theory of point estimation.

Let $(\Omega, \mathscr{A})$ and $(\mathscr{R}, \mathscr{B})$ be measurable spaces with $\mathscr{T}=R^{m}$ and with $\mathscr{B}$ the Borel field on $\mathscr{O}$. With the "parameter space" $\theta \subset R^{n}$ serving as index set, let $\left(P r_{\theta}\right)_{\theta \in \theta}$ be a family of probability measures with each defined on $(\Omega, \mathscr{N})$. Then to relate the discussion to statistical applications, let the data for some experiment be a random vector (finite real vector-valued Borel-measurable function) $X:\left(\Omega, \mathscr{A},\left(\operatorname{Pr}_{\theta}\right)_{\theta \in \theta}\right) \rightarrow$ ( $\left.\mathscr{P}, \mathscr{B},\left(P_{\theta}\right)_{\theta \in \Theta}\right)$, where $P_{\theta}$ is the probability distribution of $X$ induced on $(\mathscr{P}, \mathscr{B})$ by $\operatorname{Pr}_{\theta}$ for $\theta \in \theta$. The value of the function $X$ at a given point in $\Omega$ does not depend upon $\theta$, although the probability measures on $(\Omega, \mathscr{A})$ and on $(\mathscr{C}, \mathscr{B})$ do depend upon $\theta$. Hence we do not write $X$ as a family $\left(X_{\theta}\right)_{\theta \in \theta}$. Finally we shall let $t:\left(\mathscr{P}, \mathscr{\mathscr { T }},\left(P_{\theta}\right)_{\theta \in \theta}\right) \rightarrow(\bar{Y}, \overline{\mathcal{F}}$, $\left.\left(\bar{Q}_{\theta}\right)_{\theta \in \theta}\right)$ be a random vector with $\bar{Y} \subset \theta$, with $\overline{\mathscr{F}}$ the Borel field on $\bar{Y}$, and with $\bar{Q}_{\theta}$ the probability distribution of $t$ induced on $\bar{Y}$ by $P_{\theta}$.

Since we have not written $t$ as a family, $\left(t_{\theta}\right)_{\theta \in \theta}$, the value of the function $t$ at a given point in does not depend upon $\theta$. It is this functional independence of $\theta$ which, by definition, distinguishes the special case of a "statistic," $t$, defined on $\left(\mathscr{P}, \mathscr{B},\left(P_{\theta}\right)_{\theta \in \theta}\right)$ from the more general concept of a family of random vectors (indexed by $\theta$ ) defined on $\left(\mathscr{C}, \mathscr{B},\left(P_{\theta}\right)_{\theta \in \theta}\right)$. This independence property is required in appli- 
cations, since the value of $\theta$ is unknown, and hence no funtions of $\theta$ could be computed in practice. Hence all point estimators in statistical theory conventionally are required to be statistics. Analogously all mappings discussed below (including our set-valued estimators) will have this "statistical" property of functional independence of $\theta$.

Statisticians require their data and their statistics (including their point estimators) to be Borel measurable to assure that the probabilities they require (to construct distribution functions) will be probability measures of measurable sets. We similarly shall seek function measurability conditions on our set-valued mappings sufficient to assure measurability of those sets whose probabilities are required in set estimation.

In point estimation, we use a statistic as an estimator of $\theta$ (which has unknown value). The value of that statistic (the "estimate" or "realization of the estimator") lies in $\theta$, and we seek a statistic whose probability distribution at any $\theta \in \theta$ is such that the statistic's value will "tend to be close to" the unknown $\theta$. But for reporting purposes, the point estimate alone contains no information about the estimator's distribution. In set estimation we more explicitly seek to "estimate" $\theta$ with a random set of values in $\theta$ such that the set will contain $\theta$ with some predetermined probability. In the following section, we shall develop measure-theoretic foundations for set estimation using measurable setvalued mappings. Our approach and objectives will be analogous to those existing in the theory of point estimation.

An excellent treatise on general statistical theory is contained in Zacks [14].

4. Confidence Procedures. We shall accept the notation for our data introduced in the previous sections. In addition let $S:\left(\mathscr{B}, \mathscr{B},\left(P_{\theta}\right)_{\theta \in \theta}\right)$ $\rightarrow\left(Y, \mathscr{F},\left(Q_{\theta}\right)_{\theta \in \theta}\right)$ be a random set with $Y \subset \mathscr{P}(\theta)-\{\phi\}$ and with $Q_{\theta}$ the probability distribution of $S$ induced on $Y$ by $P_{\theta}$. Assume that $S$ is surjective. The relation of statistical confidence sets to the following definition will be investigated in this section.

Definition 5. The surjective random set $S$ is a confidence procedure, if $\mathscr{F}$ is the elementary $\sigma$-field on $Y$.

Observe that confidence procedures are an obvious extension of statistics to set-valued measurable transformations. A common property of confidence procedures now can be defined.

Definition 6. Let $S$ be a confidence procedure. Then $S$ has (lower) confidence level $\gamma=\inf \left\{Q_{\theta}\left(\mathscr{C}_{\theta}\right): \theta \in \theta\right\}$. If $S$ is a confidence procedure, and if $x \in \mathscr{B}$, then $S(x)$ will be called a confidence subset of $\Theta$. Such a confidence subset will be said to have confidence level $\gamma$ if $S$ does. 
Our confidence subsets of $\theta$ serve the purpose of the loosely named confidence sets for $\theta$ in existing statistical theory. The two theorems below demonstrate that the confidence procedures specified by Definition 5 possess the properties desired of them.

Lemma 1. Let $T:\left(\mathscr{C}, \mathscr{B},\left(P_{\theta}\right)_{\theta \in \Theta}\right) \rightarrow Y$ be a surjective mapping. Then $\{x \in \mathscr{b}: \theta \in T(x)\}=T^{-1}\left(\mathscr{C}_{\theta}\right)$ for all $\theta \in \theta$.

Proof. $\mathcal{N}_{\mathscr{T}}(\theta) \cap Y=\mathcal{N}_{\mathscr{T}}(\theta) \cap T(\mathscr{O})=\left\{U \subset R^{n}: \theta \in U\right\} \cap T(\mathscr{C})$ $=\{U \in T(\mathscr{R}): \theta \in U\}$. So $T^{-1}\left(\subset^{\prime}(\theta) \cap Y\right)=T^{-1}(\{U \in T(\mathscr{C}): \theta \in$ $U\})=\{x \in \mathscr{C}: \theta \in T(x)\}$.

Lemma 1 provides the motivation and justification for Definition 6 above, since $Q_{\theta}\left(\mathscr{C}_{\theta}\right)=P_{\theta}\left[S^{-1}\left(\mathscr{C}_{\theta}\right)\right]=P_{\theta}(\{x \in \mathscr{R}: \theta \in S(x)\})$ by Lemma 1. Similarly Theorem 1 below demonstrates that Definition 5 captures the property desired of confidence procedures, while Theorem 2, the converse of Theorem 1, demonstrates that Definition 5 requires the minimal function measurability condition sufficient to capture that property.

TheOREM 1. If $S$ is a confidence procedure, then $\{x \in \mathscr{T}: \theta \in S(x)\}$ $\in \mathscr{B}$ for every $\theta \in \theta$.

Proof. Let $\theta \in \theta$. Since $S$ is a confidence procedure, $\mathscr{F}$ is the elementary $\sigma$-field on $Y$ and is therefore generated by $\Sigma$. So $\mathscr{C}_{\theta} \in \mathscr{F}$. Hence by the measurability of $S, S^{-1}\left(\mathscr{C}_{\theta}\right) \in \mathscr{B}$. Finally by Lemma 1 , $\{x \in \mathscr{C}: \theta \in S(x)\} \in \mathscr{B}$.

TheOREM 2. Let $T:\left(\mathscr{P}, \mathscr{B},\left(P_{\theta}\right)_{\theta \in \Theta}\right) \rightarrow Y$ be a surjective set-valued mapping. Define $\mathscr{H}$ to be the smallest $\sigma$-field on $Y$ such that $\{x \in \mathscr{F}: \theta \in T(x)\} \in T^{-1}(\mathscr{H})$ for all $\theta \in \Theta$. If $T$ is such that $\{x \in \mathscr{B}: \theta \in T(x)\} \in \mathscr{B}$ for all $\theta \in \theta$, and if $Q_{\theta}$ is the probability distribution of $T$ induced on $Y$ by $P_{\theta}$, then $T:\left(\mathscr{C}, \mathscr{B},\left(P_{\theta}\right)_{\theta \in \Theta}\right) \rightarrow(Y, \mathscr{H}$, $\left.\left(Q_{\theta}\right)_{\theta \in \theta}\right)$ is a confidence procedure.

Proof. Let $\mathscr{H}_{0}$ be any $\sigma$-field on $Y$. By Lemma 1 , we know that

$$
\{x \in \mathscr{C}: \theta \in T(x)\}=T^{-1}\left(\mathscr{C}_{\theta}\right) \text { for every } \theta \in \theta \text {. }
$$

Hence it follows that

$$
\{x \in \mathscr{C}: \theta \in T(x)\} \in \mathrm{T}^{-1}\left(\mathscr{H}_{0}\right) \text { for every } \theta \in \Theta
$$

if and only if $\left\{T^{-1}\left(\mathscr{C}_{\theta}\right): \theta \in \theta\right\} \subset T^{-1}\left(\mathscr{H}_{0}\right)$. Although $T$ need not be injective, it nevertheless is true in general for $T^{-1}$ that $T^{-1}\left(N_{1}\right)=$ $T^{-1}\left(N_{2}\right)$ if and only if $N_{1}=N_{2}$. Hence $\Sigma \subset \mathscr{H}_{0}$ if and only if (ii) is true. Thus $\mathscr{H}$ exists and is the elementary $\sigma$-field on $Y$. 
Since $\{x \in \mathscr{P}: \theta \in T(x)\} \in \mathscr{\mathscr { B }}$, it follows from (i) that $T^{-1}(\Sigma) \subset \mathscr{B}$. So observing that $T^{-1}(\mathscr{H})=T^{-1}[\sigma(\Sigma)]=\sigma\left[T^{-1}(\Sigma)\right]$, we conclude that $T^{-1}(\mathscr{H}) \subset \mathscr{B}$, since $\sigma\left[T^{-1}(\Sigma)\right]$ is the smallest $\sigma$-field containing $T^{-1}(\Sigma)$. Thus $T:\left(\mathscr{Y}, \mathscr{H},\left(P_{\theta}\right)_{\theta \in \theta}\right) \rightarrow\left(Y, \mathscr{H},\left(Q_{\theta}\right)_{\theta \in \Theta}\right.$ is a confidence procedure.

In formulating Theorem 2 , we have defined $\mathscr{H}$ so that $(\mathscr{B}, \mathscr{H})$ measurability of $T$ would be as weak a condition as possible but sufficient to assure that $\{x \in \mathscr{R}: \theta \in S(x)\} \in \mathscr{B}$ for all $\theta \in \Theta$. Conditionally upon that selection of $\mathscr{H}$, Theorem 2 is the converse of Theorem 1 . The above lemma and theorems relate to the desired measurability of the set $\{x \in \mathscr{P}: \theta \in S(x)\}$ in applied statistics. However many measure-theoretic theorems on confidence procedures are stated in terms of the measure of the set $[\theta \in S(X)]$. The following lemma provides the link between the two measurability criteria and thereby will assure the measurability of $[\theta \in S(X)]$ whenever $S$ is a confidence procedure.

Lemma 2. If $\{x \in \mathscr{P}: \theta \in S(x)\} \in \mathscr{H}$, then $[\theta \in S(x)] \in \mathscr{A}$.

Proof. Let $A=\{x \in \mathscr{T}: \theta \in S(x)\}$. Then $\omega \in[X \in A]==X(\omega) \in$ $A \Rightarrow=\theta \in S(X(\omega))==\omega \in[\theta \in S(X)]$. Hence $[X \in A]=[\theta \in S(X)]$. So $[\theta \in S(X)]=X^{-1}(A)=X^{-1}(\{x \in \mathscr{X}: \theta \in S(x)\}$. Now suppose $\{x \in \mathscr{P}: \theta \in S(x)\} \in \mathscr{B}$. Then $[\theta \in S(X)] \in \mathscr{A}$, since $X$ is $(\mathscr{A}, \mathscr{B})$ measurable.

Thus, as desired, the following measurability property obtains. It follows directly from Lemma 2 and Theorem 1. Alternatively Corollary 1 could be acquired from Theorem 1 and the fact that the composition of measurable mappings is measurable.

Corollary 1 to Theorem 1 . If $S$ is a confidence procedure, then $[\theta \in S(x)] \in \mathscr{A}$ for every $\theta \in \theta$.

Although the codomain of $S$ is $Y$ rather than $\theta$, define the graph of $S$ to be $\Gamma=\{(x, \theta) \in \mathscr{F} \times \theta: \theta \in S(x), x \in \mathscr{X}\}$. Wallace [13] defines a subset of the set of confidence procedures in terms of the measurability of $\Gamma$ in the relevant product space. The following corollary demonstrates that a related although weaker measurability condition is satisfied for confidence procedures in general.

Corollary 2 to Theorem 1. Let $\Gamma$ be the graph of $S$, and let $\Gamma_{\theta}$ be the section of $\Gamma$ at $\theta \in \theta$. Then if $S$, is a confidence procedure, $\Gamma_{\theta} \in \mathscr{B}$ for every $\theta \in \theta$.

Proof. Since $\Gamma_{\theta}=\{x \in \mathscr{K}:(x, \theta) \in \Gamma\}=\{x \in \mathscr{K}: \theta \in S(x)\}$, the measurability of $\Gamma_{\theta}$ follows from Theorem 1 . 
Let $\Gamma_{x}=S(x)$ be the section of $\Gamma$ at $x$. Then observe that if $S$ is a confidence procedure, $\Gamma_{x}$ need not be a Borel measurable subset of $\theta$. Similarly a random interval $\left[\theta_{1}, \theta_{2}\right]$ can be a confidence interval for some parameter $\theta$ even if $\theta_{1}$ and $\theta_{2}$ are not random variables, although conversely if they are random variables, $\left[\theta_{1}, \theta_{2}\right]$ is a confidence procedure. Wallace's assumption that $\Gamma$ is a measurable subset of the product space $\mathscr{K} \times \theta$ identifies a useful subset of the set of confidence procedures rather than the entire set. Also observe that a set $\Gamma$ measurable in the product space $\mathscr{R} \times \theta$ is the graph of some confidence procedure only if $\Gamma_{x}$ is non-empty at all $x \in \mathscr{T}$.

5. Borel Confidence Procedures. Mappings used in statistics nearly always are Borel measurable. Borel measurability provides access to the many useful results on Borel measurable mappings and assures that our mappings will have properties conventionally expected to obtain in statistical inference. But we have not required Borel measurability of confidence procedures. In this section we shall explore conditions sufficient for confidence procedures to be Borel measurable, and we shall identify the form that will be taken by the resulting Borel measurability condition.

We introduce our definition of a Borel confidence procedure.

Definition 7. The surjective random set $S:\left(\mathscr{R}, \mathscr{B},\left(P_{\theta}\right)_{\theta \in \theta}\right) \rightarrow(Y$, $\left.\mathscr{F}_{0},\left(Q_{\theta}\right)_{\theta \in \theta}\right)$ is a Borel confidence procedure, if $\mathscr{F}_{0}$ is the elementary Borel field on $Y$.

We now shall prove the following theorem.

Theorem 3. Let the surjective random set $S:\left(\mathscr{R}, \mathscr{B},\left(P_{\theta}\right)_{\theta \in \theta}\right) \rightarrow(Y$, $\left.\mathscr{F},\left(Q_{\theta}\right)_{\theta \in \theta}\right)$ be a confidence procedure. Let $Y$ carry the subspace neighborhood system topology, and let $\mathscr{T}$ carry the ordinary (Euclidean) topo$\log y$. Let $\mathrm{S}$ be a continuous open mapping. Then $\mathrm{S}$ is a Borel confidence procedure.

Proof. The Euclidean space, $\mathscr{K}$, is $2^{\circ}$ countable. But $2^{\circ}$ countability is invariant under continuous open surjections. Hence $Y$ is $2^{\circ}$ countable, and thereby the Borel field generated by $\Sigma$ equals the $\sigma$-field generated by $\Sigma$.

In both theory and applications, it could be useful (and perhaps common) for confidence procedures to be open and continuous mappings (although the confidence subsets need not be open subsets of the Euclidean space $\theta$ ). When $S$ is continuous and open, the resulting theory of Borel confidence procedures provides an even more complete analog to the theory of statistics than did our theory of general confidence 
procedures. But Theorem 1 applies to Borel confidence procedures even when they are not continuous or open mappings. So it would be permissible for most purposes (and perhaps commonly would be advantageous) to restrict consideration to Borel confidence procedures. By doing so, we would assure immediate access to the useful properties of Borel functions (and to results requiring the range space to carry a topology).

6. Discussion. The fundamental property of a confidence procedure $S$ is the measurability of the set $[\theta \in S(X)]$, while the Borel measurability of the set $\{x \in \mathscr{B}: \theta \in S(x)\}$ is sufficient to assure the measurability of $[\theta \in S(X)]$. Now to define the set of confidence procedures $S:\left(\mathscr{C}, \mathscr{B},\left(P_{\theta}\right)_{\theta \in \Theta}\right) \rightarrow\left(Y, \mathscr{F},\left(Q_{\theta}\right)_{\theta \in \Theta}\right)$ to be a subset of the set of random sets, one must specify the $\sigma$-field $\mathscr{F}$. The analysis above specifies $\mathscr{F}$ in such a manner as to assure that the measurability of $\{x \in \mathscr{C}: \theta \in S(x)\}$ follows from the function measurability condition $S^{-1}(\mathscr{F}) \subset \mathscr{B}$. The result is that all desired set measurability conditions follow from the measurability of the mapping $S$. The analogous result for measurable functions provides the motivation for the use of statistics in point estimation. The objective in either case is to assure the measurability of sets whenever their probability measure is of interest.

Regarding the possibility of the extension of Wallace's definition solely in terms of the mappings' graphs, observe that a random set is described by a quintuple $(\mathscr{B}, \mathscr{B}, \Gamma, Y, \mathscr{F})$, while from the graph, $\Gamma$, alone one can determine only the couple $(\mathscr{C}, Y)$. In summary the anlaysis above identifies confidence procedures as simple and natural extensions of statistics and identifies the set of confidence procedures as one of the simplest subsets of the set of random sets. The motivation and method throughout are identical to those underlying the conventional use of statistics as point estimators. The necessity to assume additional ad hoc set measurability conditions is removed.

\section{REFERENCES}

1. J. Dugundji, Topology, Allyn, 1964.

2. A. Friedman, Foundations of Modern Analysis, Holt, 1970.

3. V. M. Joshi, Admissibility of Confidence Intervals, Ann. Math. Statist. 37 (1966), $629-638$.

4. - Inadmissibility of the Usual Confidence Sets for the Mean of a Multivariate Normal Population, Ann. Math. Statist. 38 (1967), 1868-1875.

5. - Admissibility of the Usual Confidence Sets for the Mean of a Univariate or Bivariate Normal Population, Ann. Math. Statist. 40 (1969), 1042-1067.

6. _ _ Admissibility of Invariant Confidence Procedures for Estimating a Location Parameter, Ann. Math. Statist. 41 (1970), 1568-1581.

7. __ Admissibility of the Usual Confidence Sets for a Class of Bivariate Populations, Ann. Math. Statist. 42 (1971), 622-679. 
8. D. G. Kendall, Founations of a Theory of Random Sets, in Stochastic Geometry, E. F. Harding and D. G. Kendall, ed., Wiley, 1974.

9. G. Matheron, Random Sets and Integral Geometry, Wiley, 1975.

10. E. Michael, Topologies on Spaces of Subsets, Trans. Amer. Math. Soc. 71 (1971), $152-182$.

11. W. Rogosinski, Volume and Integral, Wiley, 1952.

12. C. Stein, Confidence Sets for the Mean of a Multivariate Normal Distribution, J. Roy. Statist. Soc. 24 (1962), 265-269.

13. D. L. Wallace, Conditional Confidence Level Properties, Ann. Math. Statist. 30 (1959), 864-876.

14. S. Zacks, The Theory of Statistical Inference, Wiley, 1971.

Division of Research and Statistics, Board of Governors of the Federal Reserve SySTEM, Washington, D.C. 20551 
